\title{
Rationalisiert IT die Ärzte weg?
}

\section{Interview mit Herrn Professor Dr. Michael Forsting}
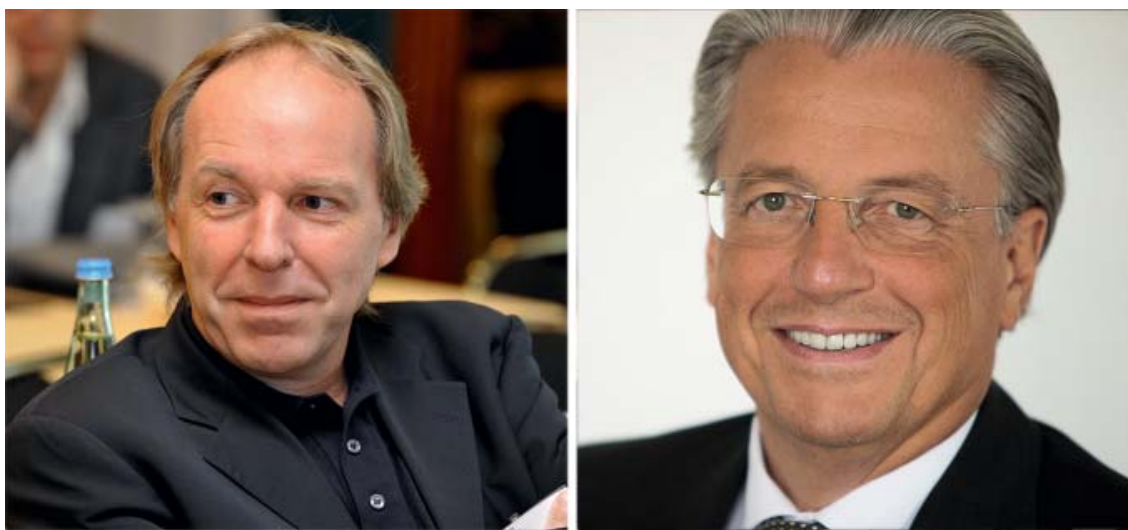

Der Kongress „ETIM 2017 - Artificial intelligence and bioprinting“ findet statt unter der Leitung von Prof. Michael Forsting (links), Direktor des Instituts für Diagnostische und Interventionelle Radiologie des UK Essen, und Prof. Jochen Werner (rechts), Ärztlicher Direktor des Universitätsklinikums Essen.

In Essen findet am 10. und 11. Februar 2017 die internationale Veranstaltung „ETIM 2017 - Artificial intelligence and bioprinting" statt (https://etim. uk-essen.de). Im Gespräch erläutert Michael Forsting die Hintergründe zu dieser Veranstaltung.

Herr Prof. Forsting, Sie veranstalten gemeinsam mit Herrn Professor Jochen Werner - dem Ärztlichen Direktor des UK Essen - am 10. und 11. Februar 2017 in Essen den ETIM-Kongress. Wofür steht ETIM und was wird auf diesem Kongress gezeigt?

ETIM steht für „Evolving technologies in medicine“. Wir haben hochkarätige Referenten aus dem In- und Ausland eingeladen, die zu den Themen „Künstliche Intelligenz im Krankenhaus“ und „3 D- Druck in der Medizin“ reden werden. Beide Bereiche haben das Potential, die Medizin innerhalb von kurzer Zeit dramatisch zu verändern. Wenn man glaubt, dass 3D-Druck in der Medizin sich darauf beschränkt, individuell angepasste Hüftprothesen herzustellen oder vielleicht noch Pathologie-adaptierte Gefäßprothesen, hat das Potential der Methode völlig verkannt. An einigen Instituten in der Welt werden bereits Lebersegmente gedruckt, an denen die Pharmaindustrie erste toxikologische Tests durchführt. Und Systeme, die mit künstlicher Intelligenz arbeiten, haben auch nicht mehr viel zu tun mit den schon lange existierenden CAD-Systemen. Sie lernen selber und vergessen auch seltene Erkrankungen nie.

Gibt es denn schon Anwendungen von Künstlicher Intelligenz in der Medizin?

Die ersten Anwendungen kommen jetzt. So gibt es ein Programm, dass automatisch die Schwere des Schlaganfalls im frühen CT mit einem etablierten Score bestimmt. Selbst sehr gute Neuroradiologen schaffen das nicht mit der Genauigkeit. Und im Unterschied zu „menschlich“ programmierten Algorithmen steckt in diesem Programm ein „deep learning“ Algorithmus, so dass das System mit validierten Datensätzen selber gelernt hat und weiter lernt. Wir selber haben mit einem solchen Algorithmus ein System auf Lungenfibrosen trainiert und auch schon mit wenigen Lernzyklen erreicht, dass der Computer die Diagnose besser stellen kann als ein Arzt.

Warum gibt es denn bisher so wenig „Big Data“-Anwendungen in der Medizin?

Der Irrtum ist, dass wir mit „Big Data“ wirklich weiter kommen. Das Hauptproblem in der Medizin sind valide Datensätze. Wenn wir als Beispiel CT-Bilder neh- men: natürlich gibt es davon Millionen Datensätze, aber um ein System lernen zu lassen, muss die Diagnose bekannt und richtig sein. Und diese Datensätze haben wir einfach nicht. Um künstliche Intelligenz in der Diagnostik ans Laufen zu bekommen, brauchen wir ganz andere Datenstrukturen und die müssen wir jetzt schnell aufbauen. Und dann wird man auch schnell Anamnesebögen mit künstlicher Intelligenz haben.

Rationalisieren wir dann mit 3D-Druck und Künstlicher Intelligenz einen Teil der Ärzte weg?

Beide Technologien werden sich nur durchsetzen, wenn dadurch die Medizin besser wird, also Implantate besser passen, länger halten, Katheter schneller in das Aneurysma manövriert werden können und Diagnosen besser und schneller gestellt werden können. Die Handlungshoheit wird aber immer ein Arzt haben. Das ist wie im Flugzeug: Eigentlich kann der Autopilot $90 \%$ der Flugreise alleine bewältigen, aber der Pilot hat immer die Handlungshoheit. Andererseits sorgt die demografische Entwicklung gerade dafür, dass immer mehr ältere Menschen durch eine gleichbleibende oder gar abnehmende Zahl von Ärzten versorgt werden müssen. Und auch das wird nur funktionieren, wenn wir Assistenzsysteme bekommen, die uns erlauben die richtige Diagnose schneller zu stellen und auch schneller die richtige Therapie einzuleiten. Insofern mache ich mir um den Arztberuf keine Sorgen.

Ist der Kongress jetzt eher etwas für ITler oder für Mediziner?

Beide Berufsgruppen sollen kommen. Bislang gibt es keine Plattform, auf der Mediziner und ITler die möglichen Anwendungen von Künstlicher Intelligenz und 3D-Druck in der Medizin besprechen können. Wir sprechen sogar noch verschiedene Sprachen. Die einen haben coole Algorithmen, die anderen kennen die medizinischen Probleme. Die einen brauchen Daten, die anderen haben sie. Und keiner durchschaut die rechtlichen Rahmenbe- 


\section{UKM}

Universitätsklinikum

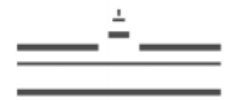

WeStFăLISCHE WILHELMS-UNIVERSITÄT Münster

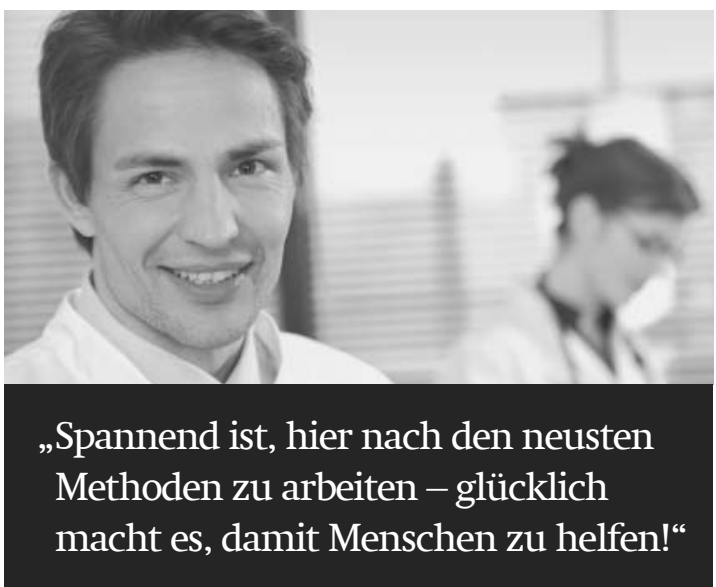

Das Universitätsklinikum Münster ist eines der führenden Krankenhäuser Deutschlands. Eine solche Position erlangt man nicht nur durch Größe und medizinische Erfolge. Wichtig ist das Engagement jedes Einzelnen. Wir brauchen Ihr Engagement, um selbst im Kleinen Großes für unsere Patienten leisten zu können. Dafür bieten wir Ihnen viele Möglichkeiten, damit Sie selbst weiter wachsen können.

Das Institut für Klinische Radiologie sucht zum nächstmöglichen Zeitpunkt zunächst befristet auf 5 Jahre mit der Möglichkeit der Verlängerung einen

\section{Neuroradiologen in Oberarztposition $(\mathrm{w} / \mathrm{m})$}

\section{oder einen}

\section{Facharzt für Radiologie (w/m)} mit Expertise auf dem Gebiet der Neuroradiologie vollzeitbeschäftigt, Vergütung nach TV-Ä, Kennziffer 12304

Das Institut für Klinische Radiologie versorgt alle stationären und ambulanten Patienten des UKM mit dem vollständigen Spektrum radiologischer Leistungen. Integraler Bestandteil des universitären Zentralinstituts ist die Neuroradiologie. Tätigkeitsschwerpunkte sind der systematische Ausbau innovativer Bildgebung bei neuroonkologischen und neuroinflammatorischen Erkrankungen sowie die interventionelle Neuroradiologie.

Ihre Perspektiven:

- Sofern noch nicht vorhanden eine vollständige Weiterbildung im Schwerpunkt Neuroradiologie mit besonderem Fokus auf die dazugehörigen Interventionen (komplettes Spektrum)

- Möglichkeit zur Habilitation sowie systematische Vorbereitung auf eine Führungsposition durch Teilnahme an UKM-Managementkursen

Wir wünschen uns von Ihnen fundierte Kenntnisse und Fertigkeiten in Neuroradiologie mit dem Schwerpunkt Schnittbildgebung sowie die Bereitschaft zu patientenorientiertem Engagement und enger Kooperation mit unseren klinischen Partnern, Forschungsinteresse und die Bereitschaft zur Unterstützung von Lehrveranstaltungen.

Bei Rückfragen wenden Sie sich bitte an Univ.-Prof. Dr. med. W. Heindel, Tel.: 0251/83-47301, E-Mail: heindel@uni-muenster.de

Wir freuen uns auf Ihre Bewerbung unter Angabe der Kennziffer bis zum 28.02.2017. Mehr Informationen erhalten Sie unter: www.karriere.ukmuenster.de

Das UKM unterstützt die Vereinbarkeit von Beruf und Familie und ist daher seit 2010 als familienbewusstes Unternehmen zertifiziert. Es besteht grundseit 2010 als familienbewusstes Unternehmen zertifiziert. Es besteht grundFrauen wird begrüßt: im Rahen der gesetzlichen Vorschriften werden Frauen bevorzugt eingestellt. Schwerbehinderte werden bei gleicher Eignung besonders berücksichtigt. dingungen. Die werden wir bei dem Kongress auch diskutieren.

\section{Aber für die Medizin ist schon die Radio- logie das Hauptanwendungsgebiet, oder?}

Ganz und gar nicht. Künstliche Intelligenz wird für den Allgemeinmediziner in der Landpraxis mindestens genauso wichtig werden wie für den Superspezialisten in der Onkologie. Radiologie ist halt schon eine digitale Disziplin, daher werden die neuen Diagnostiksysteme hier wohl am schnellsten zum Einsatz kommen.

\section{Wo meldet man sich an?}

Wir haben eine Website und man kann sich natürlich online anmelden (https:// etim.uk-essen.de). Damit möglichst viele kommen, nehmen wir auch keine Kongressgebühr. 